\title{
Some conceptual issues in the origin of eusociality
}

\author{
J. W. Stubblefield and \\ E. L. Charnov
}

Department of Biology, University of Utah, Salt Lake City, UT 84112, U.S.A.

Certain issues arising in connection with the evolutionary origins of eusociality are discussed. Previous results about when natural selection favours helping behaviour are generlised, and the differing viewpoints of both parents and offspring are considered. Particular attention is given to the evolutionary implications of different patterns of overlapping generations observed in bivoltine insects. As argued by Seger (1983), these patterns imply different conditions under which a daughter is selected to help her mother rear additional siblings in haplodiploid populations. Other factors that can alter the selective advantages of helping behaviour under haplodiploidy are also discussed, including the possibility of sex ratio manipulation and the novel result that helping behaviour may be locally favoured in populations that are spatially patchy with respect to sex-specific fitness. A new hypothesis is also presented: The fact that sisters are selected to aid their mother to parasitise other sisters may have played an important role in the origins of eusociality. A given offspring benefits from having maternally parasitised siblings because such siblings rear additional siblings (to which the given offspring is more closely related) instead of nieces and nephews. Finally, the importance of haploidiploidy in the origins of eusociality is discounted; the virtually unique biology of aculeate Hymenoptera would seem to be of much greater importance.

Eusocial behaviour such that offspring aid their parents (or mother) to rear their siblings, is found in a few vertebrates, termites, and some Hymenoptera. Four questions have dominated recent thinking on the origin and maintenance of eusociality. First, what life history conditions (e.g., seasonality) favour some individuals to become workers? Second, what is the special role of haplodiploidy, if any? Third, did the initial stages of eusociality reflect the interests of parents or offspring or both? Fourth, how are parent-offspring conflicts resolved in existing eusocial societies? Recent reviews by Brockman (1984) and Andersson (1984) should be consulted for up to date discussions of earlier work. This note discusses two important considerations in the origins of eusocial behavior: life history conditions and parent versus offspring interests. Our purpose is to point out some potentially important factors for the evolution of eusociality.

We first describe a typical life history for a solitary wasp or bee. Consider a female that emerges in the spring and begins constructing cells. In each cell, she lays an egg and provides it with sufficient food to develop into an adult. If some of her offspring mature while she is still alive and reproducing, these offspring may be considered to have two options. In the case of daughters at least, they can mate and rear their own sons and daughters, or they can help their mother rear additional sibs. Subsocial nesting in which mated daughters return to produce their own offspring in or near the maternal nest provides a likely starting point for eusociality to evolve. Subsocial nesting may arise for a variety of purely selfish reasons including reduced losses to parasites and reduced nest construction costs. In any event, the essential ingredients are a population with overlapping generations and the options of personal reproduction or helping available to offspring produced early in the year.

As recently noted by Seger (1983), there are two patterns of overlapping generations observed in bivoltine wasps and bees, and these have different implications for the origin of eusociality. These two patterns, which we shall refer to as the larval diapause and female hibernation cases, are illustrated in figs. 1 and 2. In the larval diapause case, second generation offspring of both sexes overwinter as virgins (usually as larvae) and do not mate until the following year. In the female 


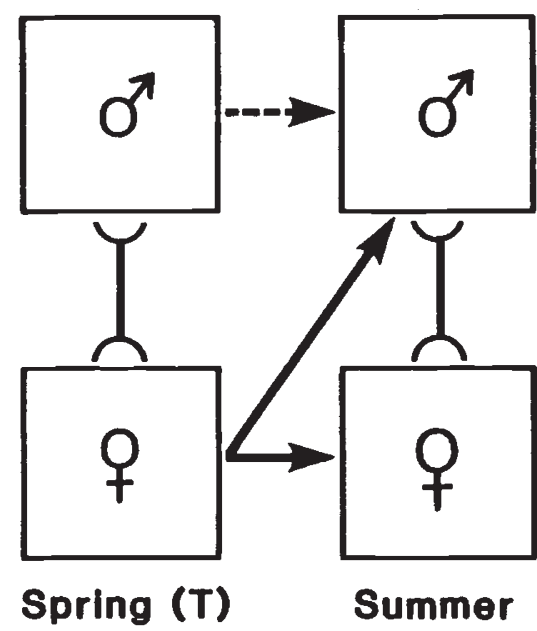

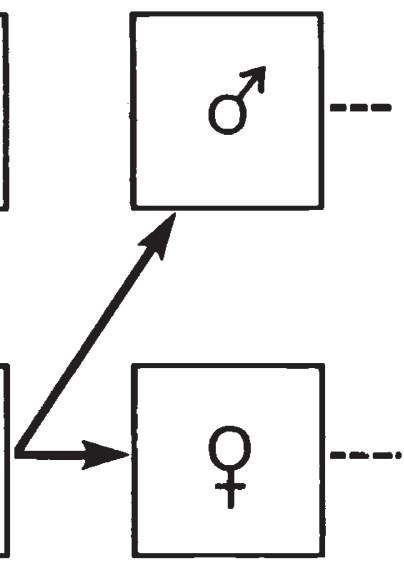

Fall (larva)

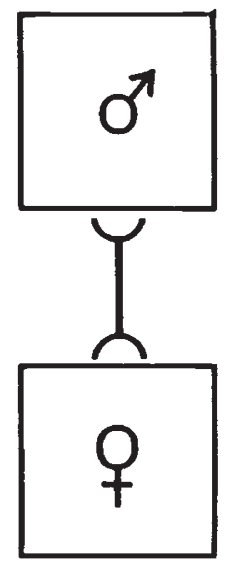

Spring $(T+1)$ (Adults)

\section{LARVAL DIAPAUSE}

Figure 1 The larval diapause pattern of overlapping generations in a bivoltine population. Two generations are produced each year, a summer generation that matures and reproduces within a single year and a winter generation that does not reproduce until the following year. Individuals of both sexes overwinter as virgins (usualy as diapausing larvae) and do not mate until the following year. Winter males mate with females in their own generation and may also survive to mate with summer females (dotted arrow). When this occurs, selection favours a female-biased sex ratio in the summer generation and a male-biased sex ratio in the winter generation. In the haplodiploid case, this overlap of generations on the male side also has the effect of raising the helping threshold for a summer daughter.

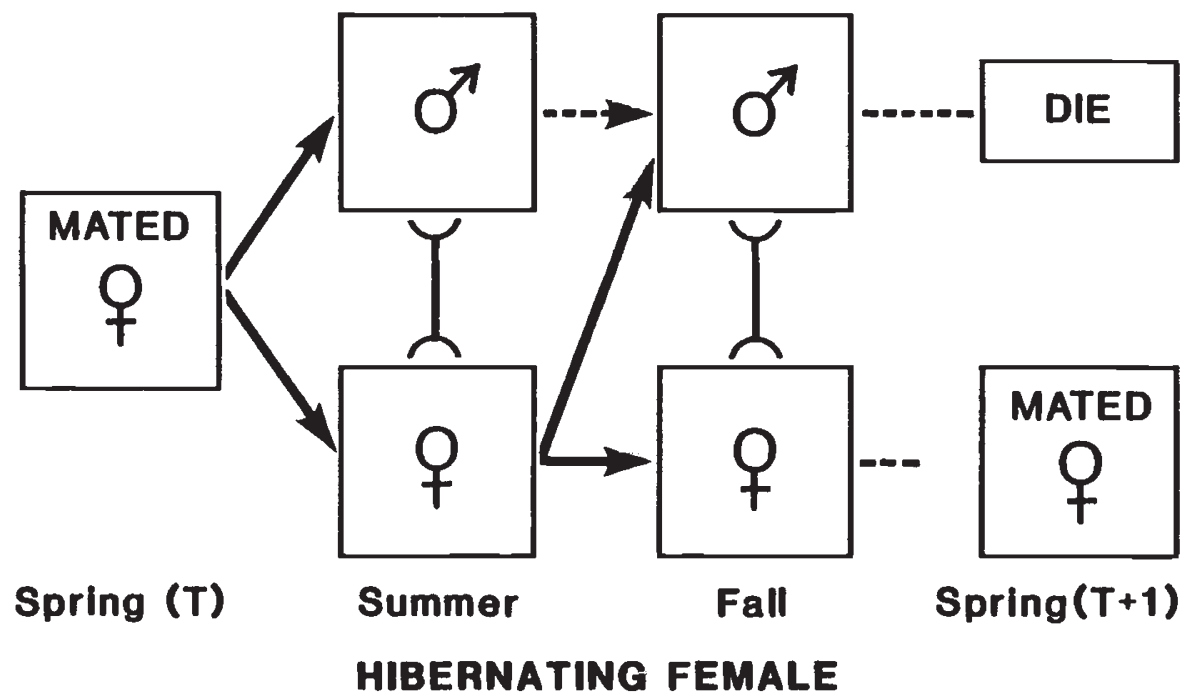

Figure 2 The female hibernation pattern of overlapping generations in a bivoltine population. In contrast to the larval diapause case, the winter generation undergoes direct development and mates in the late summer or fall, and only mated females survive to the following year. In this case, it is the summer males that may survive to enter a second breeding population (dotted arrow), and when this occurs selection favours a male-biased sex ratio in the summer generation and a female-biased sex ratio in the winter generation. In the haplodiploid case, this overlap of generations on the male side also has the effect of lowering the helping threshold for a summer daughter.

hibernation case, on the other hand, the second generation mates in the late summer or fall, and only mated females survive to the following year. In both cases, males in the first breeding population in a given year may survive to mate again in the second breeding population, as indicated by the dotted arrows in the figures, and this is the critical factor for the selective advantages of helping behaviour. In the larval diapause case, it is winter males that enter two breeding popula- 
tions, and this has the effect of making winter females less valuable relative to winter males. In the female hibernation case, however, it is summer males that enter two breeding populations, and this has the effect of making winter females more valuable relative to winter males. These shifts in the relative value of winter males and females alters the selective value of helping behaviour in haplodiploid populations, as Seger (1983) was first to recognise.

From genetic models already present in the literature together with some we have recently developed ourselves, it is now possible to present a fairly detailed picture of the selective tradeoffs involved in the origin of eusociality, and we propose to discuss the central results of this formal modeling here. We shall focus on two complementary pathways by which sociality may arise, the willing worker case and the parasitic parent case. In the case of a willing worker, we are concerned with the conditions under which a summer offspring (or potential worker) gains a selective advantage by helping her mother (a winter female or potential queen) to rear additional sibs at the expense of producing fewer offspring of her own. In the case of a parasitic parent, on the other hand, we are concerned with the conditions under which a winter female may gain a selective advantage by parasitising the efforts of her summer offspring even if her offspring's fitness is reduced by doing so. Some authors have emphasised one of these possibilities to the virtual exclusion of the other, but it is clear that they are complementary aspects of the same situation and should both be considered. The willing worker case emphasises the offspring point of view, while the parasitic parent case emphasises the parental point of view.

We assume that females mate only once, and consider a panmictic bivoltine population which niay exhibit either the larval diapause or the female hibernation pattern. For the purposes of this note, we shall also adopt a few simplifying assumptions that do not alter the qualitative conclusions we wish to emphasise. In particular, we assume that (1) the winter offspring produced by the two kinds of female share the same sex-specific fitnesses; and (2) winter females produce no winter offspring prior to the time when helping behaviour first arises (i.e., Seger's $T=0$ ). A general treatment that relaxes these simplifying assumptions is provided in Stubblefield (1985) which may also be consulted for mathematical details omitted here.

A convenient way to express the conditions under which helping behaviour gains a selective advantage is in terms of the ratio between the number of offspring by which the reproductive output of a potential queen is increased and the number of offspring by which the output of a daughter is decreased if she becomes a helper. Let $\Delta n_{q}$ and $\Delta n_{w}$ be the numbers of offspring gained and lost in this way and define,

$$
H=\frac{\Delta n_{q}}{\Delta n_{w}}
$$

as the ratio between them. Under haplodiploidy, the tradeoffs differ between female $(f)$ and male $(m)$, offspring, and we also define

$$
H_{f}=\frac{\Delta f_{q}}{\Delta f_{w}} \quad \text { and } \quad H_{m}=\frac{\Delta m_{q}}{\Delta m_{w}}
$$

where $\left(\Delta f_{q}, \Delta m_{q}\right)$ is the amount by which the output of the mother is increased and $\left(\Delta f_{w}, \Delta m_{w}\right)$ is the amount by which the output of a helping daughter is decreased. As noted above, the fact that early males may enter two breeding populations has important implications for the selective advantages of helping behaviour, and we define $Z$ as the fraction of all males in the second breeding population that survived from the first. For each case we may wish to consider, there is some minimal or threshold value of $H$ (or $H_{f}$ and $H_{m}$ ) that must be exceeded if the party under consideration is to gain a fitness benefit from helping behaviour, and we express these threshold conditions as functions of $Z$.

Let us first consider the willing worker case under haplodiploidy. In this case, a summer daughter that becomes a helper gains $\left(\Delta f_{q}, \Delta m_{q}\right)$ additional sibs to which she is related by $(3 / 4$, $1 / 4)$ at the expense of producing $\left(\Delta f_{w}, \Delta m_{w}\right)$ fewer offspring of her own, to which she is related by $(1 / 2,1 / 2)$, and gains a selective advantage by becoming a helper if

$$
H>1+\frac{Z}{2+Z}
$$

in the larval diapause case, and

$$
H>1-\frac{Z}{2+Z}
$$

in the female hibernation case. When $Z=0$, we have $H>1$ so that a daughter must be able to increase the output of her mother by more than she decreases her own in order to gain a selective advantage from becoming a helper. All models prior to Seger (1983) considered this case. As noted by Seger, however, the threshold is raised above 
unity in the larval diapause case and lowered below unity in the female hibernation case when $Z>0$. Most solitary wasps and bees exhibit the larval diapause pattern, and we may conclude that they are unlikely candidates for evolving sociality by the willing worker route. The only solitary species known to exhibit the female hibernation pattern are some halictine bees (Seger, 1983; Brockmann, 1984), and the reduced threshold in this case may have been a significant factor in the origin of sociality in this group of bees which is well known for its diversity of social behaviour. The female hibernation pattern is the typical situation in temperate social Hymenoptera today, but one can only speculate whether or not this was true when sociality first arose in each group.

It should be noted that the thresholds given here do not require that females employ their optimal sex ratios but only that summer and winter females employ the same winter sex ratio whatever it may be. Females in populations with $Z>0$ are selected to employ biased sex ratios (Seger, 1983; Stubblefield, 1985), but whether or not they do employ such sex ratios is not the critical factor for an altered helping threshold. Instead, the critical factor is that the expected contributions to future generations differ between winter females and winter males. If the fitness of winter females is reduced relative to winter males, as arises in the larval diapause case, with $Z>0$, the helping threshold is raised above unity, and if the fitness of winter females is increased relative to winter males, as arises in the female hibernation case with $Z>0$, the helping threshold is lowered below unity. We further suggest that any other factor that increases the fitness of winter females relative to winter males would also give a reduced helping threshold. One class of models with the required fitness shifts assumes a panmictic population that is subdivided into a number of patches some of which are relatively better for female production and others relatively better for male production (see Charnov, $1979 ; 1982)$. In this case, we expect a reduced threshold in female-preferred patches and an increased threshold in male-preferred patches. Using a single locus model with $Z=0$, we have confirmed that gene for helping behaviour spreads rapidly in a spatially patchy population if it is expressed mostly in female-preferred patches. We suggest as a general principle that any local conditions that favour a female-biased sex ratio also have the effect of reducing the helping threshold below unity in haplodiploid populations, provided worker behaviour is expressed mostly in / at female preferred places or times.
As widely recognised after the seminal papers of Hamilton $(1964 ; 1972)$, the asymmetric degrees of relatedness under haplodiploidy provide a selective advantage for helping behaviour if daughters are somehow able to trade daughters for sisters more than they trade sons for brothers. In particular, we have

$$
H_{f}>2 / 3 \text { and } H_{m}>2
$$

for any value of $Z$. Consequently, the willing worker threshold is less than unity if the resources transferred from a helping daughter to her mother are invested relatively more in sisters than they would have been in daughters (i.e., $\Delta f_{1} / \Delta m_{q}>$ $\left.\Delta f_{w} / \Delta m_{w}\right)$. Since there is no a priori reason for queens to produce a more female-biased sex ratio, daughters must be able to alter their mother's sex ratio in order to benefit from a reduced threshold. Since sex-biased helping has received a great deal of attention particularly after Trivers and Hare (1976) presented data that suggested that workers might control the sex ratio in social haplodiploids. We are skeptical about the importance of sexbiased helping as a factor in the origin of eusociality for several reasons. In the first place, it seems unlikely to us that daughters could control their mother's sex ratio, particularly in the earliest stages of social evolution (Charnov, 1978). It is sometimes suggested that workers are in a unique position to influence investment between the sexes because it is they who actually care for the developing reproductives. The difficulty with this argument is that, although workers are selected to invest more of the colony's resources in each female reproductive and less in each male one than would be preferred by the queen and are even selected to kill some of the queen's sons, the queen is simultaneously selected (given $Z=0$ ) to alter her sex ratio in such a way as to achieve equal investment in the two sexes, and we do not see how the workers could force her to do otherwise. Secondly, even if we grant workers the ability to control the queen's sex ratio (which we doubt), the expected outcome is a situation in which the queen produces all the females while the workers produce all the males. To go any further than this, the workers would be trading sons for brothers, and the threshold $H_{m}>2$ comes into play. Iwasa (1981) has argued that workers have sometimes become so efficient at rearing the queen's offspring that this threshold has been surpassed so that it is in the interest of workers to allow the queen to produce males. We are skeptical about this argument because the required doubling of efficiency seems unlikely and because any efficiency gains that 
might be achieved would seem to apply just as well to producing the worker's sons as those of the queen. It is possible that sex-biased helping played some role in the earliest stages of social evolution, but in our view its importance has been exaggerated by some of authors.

The willing worker case under diploidy is much simpler. In this case, a helping offspring gains additional sibs to which it is related by $(1 / 2,1 / 2)$ at the expense of producing fewer offspring to which it is also related by $(1 / 2,1 / 2)$, and we have

$$
H, H_{f}, H_{m}>1
$$

for any value of $Z$. None of the special conditions that serve to reduce the threshold under haplodiploidy apply under diploidy, and an offspring must be able to increase the reproductive output of its mother by more than it reduces its own in order to gain a selective advantage from becoming a helper. It is now well known that the willing worker threshold is $H>1$ under both forms of inheritance when $Z=0$ and there is no sexbiased helping (Craig, 1979; Kasuya, 1981), but it does not appear to be widely recognised that $H>1$ is a necessary condition for a sterile worker strategy to increase to fixation under haplodiploidy even if the initial threshold is reduced because of one of the factors discussed above. In the case of sexbiased helping, helping behaviour spreads under a reduced threshold only up to the point where the queen produces all females and the workers produce all males. In the female hibernation case with $Z>0$, the threshold approaches unity as helping spreads since $Z$ is expected to decrease because it is more advantageous for a winter female to produce summer daughters that become helpers rather than summer sons who do not. Similarly, in the model of spatial patchiness mentioned above, helping spreads under a reduced threshold in female-preferred patches but not male-preferred patches. Perhaps the most important lesson to be drawn from available results on the willing worker threshold is that offspring may often be selectively indifferent as to whether they become workers or not (Charnov, 1978) and that we should now turn our attention away from the most general features of genetic systems in order to investigate those biological details that may tip the balance in favour of sociality.

So far we have considered only the benefits that may accrue to an offspring by becoming a helper, and we have yet to consider the situation from the parental point of view. In what we have called the parasitic parent case, a mother (or a diploid father) gains additional offspring to which she is related by $(1 / 2,1 / 2)$ at the expense of having fewer grandoffspring to which she is related by $(1 / 4,1 / 4)$, and hwe have

$$
H>1 / 2
$$

for both the larval diapause and the female hibernation cases with any value of $Z$. This result applies to both diploidy and haplodiploidy, and we see that a mother stands to gain a substantial selective advantage if she can somehow divert resources from the production of grandoffspring to the production of additional offspring. This was first developed in Charnov (1978). In the case of wasps and bees, a potential queen could do this by replacing some of her daughter's eggs with some of her own. In this way, a potential queen effectively steals the resources that a daughter provides for her own offspring. Behaviour of this sort, referred to as cleptoparasitism, has arisen repeatedly in the aculeate Hymenoptera where it is typically another species that is parasitised, but cleptoparasitism may also arise within a single species. Certainly, a mother is under strong selection to parasitise her daughters if she has the opportunity to do so. She may also gain an additional advantage by eating her daughter's eggs and thus gain high quality nutrients which may permit her to lay additional eggs, and we remark that a female who finds herself in a position to be a parasitic parent is under strong selection to increase her longevity and fecundity in order to exploit the advantages of parasitism. There may also be an asymmetry of opportunity between parasitic parents and resisting offspring in subsocial nests where parental parasitism seems most likely to arise. In particular, a reproductive daughter must often leave her own cells unguarded in order to make repeated trips away from the nest in order to forage for food for herself and her offspring and perhaps also for nesting material, while a parasitic mother has only to feed herself and search for opportunities to substitute her eggs for those of her daughters. Moreover, a daughter has no incentive to resist maternal parasitism unless the willing worker threshold is raised above unity as arises in the larval diapause case. The sizeable gains obtained by parasitic parents combined with asymmetric opportunity and indifferent offspring makes the parasitic parent pathway a plausible avenue by which insect sociality could have arisen.

A further consideration arising in the parasitic parent case has been suggested by Stubblefield (1985) and has to do with the benefits that accrue 
to daughters if their sisters are subjected to parental parasitism. In the haplodiploid case, a daughter with a parasitised sister gains additional sibs to which she is related by $(3 / 4,1 / 4)$ at the expense of having fewer nieces and nephews to which she is related by $(3 / 8,3 / 8)$, and such a daughter gains a selective advantage if

$$
H>1-\frac{1-Z}{4+2 Z}
$$

in the larval diapause case, and if

$$
H>1-\frac{1+2 Z}{4+2 Z}
$$

in the female hibernation case. When $Z=0$, we have $H>3 / 4$ in both cases, and the threshold is less than unity for any intermediate value of $Z$. Similarly, in the diploid case, an offspring of either sex gains additional sibs to which it is related by $(1 / 2,1 / 2)$ at the expense of having fewer nieces and nephews to which it is related by $(1 / 4,1 / 4)$, and an offspring with a parasitised sibling gains a fitness benefit if

$$
H>1 / 2
$$

for any value of $Z$. Several conclusions would seem to follow from these results. In the first place, we do not expect daughters to guard each other's eggs from parental parasitism. Indeed, offspring are selected to actively encourage parental parasitism of their siblings. It would be in their interest, for example, to provide food to their mother if this resulted in greater parental parasitism of siblings. This suggests that some form of food transfer from daughters to mothers may have arisen very early in the evolution of hymenopteran societies. Instead of sharing a common interest to resist maternal parasitism, daughters are divided among themselves to the extent that each of them is allied with the mother against all of the others. This factor could have played a significant role in the early stages of social evolution, and helps to explain why food transfer is a general feature of hymenopteran societies.

The purpose of this discussion has been to provide a concise statement of how we now view the problem of social origins, and we admit to having passed over a number of relevant details. We applaud the trend evident in recent reviews to explore the diversity of factors that may have contributed to social evolution rather than putting too much emphasis on only one aspect of the problem. In our view, some combination of parasitic parents and willing, or at least indifferent, workers provide the most likely scenario for the origin of insect societies, particularly when combined with the advantages accruing to daughters that aid their mother to parasitise their sisters. If this view is largely correct, it remains unexplained why eusociality has arisen repeatedly in the haplodiploid Hymenoptera but only once in diploid insects, a remarkable fact that has done much to fuel enthusiasm for the idea that haplodiploidy is particularly favourable for social evolution. We consider it just as remarkable, however, that all social haplodiploids belong to the aculeate Hymenoptera and none to the much larger number of other haplodiploid insects including the vast numbers of parasitic Hymenoptera. This suggests that we should look to the special features of aculeate wasps and bees rather than haplodiploidy in order to find an explanation for the repeated origins of eusociality. Fortunately, we do not have to look very far. Aculeate wasps and bees are, for example, virtually unique among insects in exhibiting advanced parental care that involves repeated trips from the nest to bring back food for the offspring, a way of life that is retained even in advanced social species. Such a form of offspring care makes parental parasitism especially easy; the simple replacement of one egg for another (mom's egg for her daughter's egg) turns a daughter into a worker, since the daughter simply feeds the offspring in her own cell. Aculeates are also remarkable in possessing a powerful string that could serve as a potent defence against predators that might find a tempting target in an unguarded colony. These and other natural historical factors may help to account for the repeated origins of eusociality in the aculeate Hymenoptera have been ably discussed by Evans (1977). We would go farther and suggest that if the wasp and bee way of life had been widely adopted by diploids, we would see multiple lineages of social diploids.

Acknowledgments This work was supported by a grant from the U.S. National Science Foundation.

\section{REFERENCES}

Andersson, M. 1984. The evolution of eusociality. Ann. Rev. Ecol. Syst., 15, 165-189.

BROCKMANN, H. J. 1984. The evolution of social behavior in insects. In Krebs, J. R. and Davies, N. B., eds., An Introduction to Behavioral Ecology, 2nd edition. Oxford: Blackwell.

CHARNOV, E. L. 1978. Evolution of eusocial behavior: offspring choice or parental parasitism? Jour. Theor. Biol., 75, 451465 . 
CHARNOV, E. L. 1979. The genetical evolution of patterns of sexuality: Darwinian fitness. Amer. Natur., 113, 465-480. CHARNOV, E. L. 1982. The Theory of Sex Allocation. Monographs in Pop. Biol. 18. Princeton University Press.

CRAIG, R. 1979. Parental manipulation, kin selection, and the evolution of altruism. Evolution, 33, 319-334.

EVANS, H. E. 1977. Extrinsic versus intrinsic factors in the evolution of insect sociality. Bioscience, 27, 613-617.

HAMILTON, W. D. 1964. The genetical evolution of social behaviour. I and II. $J$. Theor. Biol, , 7, 1-52.

HAMILTON, W. D. 1972. Altruism and related phenomena, mainly in social insects. Ann. Rev. Ecol. Syst., 3, 193-232.
IWASA, Y. 1981. Role of sex ratio in the evolution of eusociality in haplodiploid social insects. $J$. Theor. Biol., 93, 125-142. KASUYA, E. 1982. Factors governing the evolution of eusociality through kin selection. Res. Popul. Ecol., 24, 174-192.

SEGER, J. 1983. Partial bivoltinism may cause alternating sex-ratio biases that favour eusociality. Nature, 301, 5962.

STUBBLEFIELD, J. w. 1985. Sex allocation in bivoltine populations: Unbeatable investment ratios and implications for the origin of eusociality. J. Theor. Biol. (in press).

TRIVERS, R. L. AND HARE, H. 1976. Haplodiploidy and the evolution of social insects. Science, 191, 249-263. 